\title{
Minerals and Bone Mineral Density in Inflammatory Bowel Diseases
}

\author{
Created by: Alicja Ewa Ratajczak ${ }^{1}$, Anna Maria Rychter ${ }^{2}$, Agnieszka $^{2}$ A \\ Zawada $^{3}$, Agnieszka Dobrowolska ${ }^{4}$, Iwona Krela-kaźmierczak 5 \\ 1, Department of Gastroenterology, Dietetics and Internal Diseases, Poznan \\ University of Medical Sciences, Poland; alicjaewaratajczak@gmail.com \\ 2, Department of Gastroenterology, Dietetics and Internal Diseases, Poznan \\ University of Medical Sciences, Poland; a.m.rychter@gmail.com \\ 3, Department of Gastroenterology, Dietetics and Internal Diseases, Poznan \\ University of Medical Sciences, Poland; aga.zawada@gmail.com \\ 4, Department of Gastroenterology, Dietetics and Internal Diseases, Poznan \\ University of Medical Sciences, Poland; agdob@ump.edu.pl \\ 5, Department of Gastroenterology, Dietetics and Internal Diseases, Poznan \\ University of Medical Sciences, Poland; krela@op.pl
}

Version received: 10 June 2020

The chronic character of inflammatory bowel diseases, such as Crohn's disease and ulcerative colitis, results in various complications. One of them is osteoporosis, manifested by low bone mineral density, which leads to an increased risk of fractures. The aetiology of low bone mineral density is multifactorial and includes both diet and nutritional status. Calcium is the most often discussed minerals with regard to bone mineral density. Moreover, phosphorus; magnesium and sodium are also involved in the formation of bone mass. Patients suffering from inflammatory bowel diseases frequently consume inadequate amounts of the aforementioned minerals or their absorption is disturbed, resulting innutritional deficiency and an increased risk of osteoporosis.

\section{Calcium and Phosphate}

Calcium (Ca) is responsible for proper inflammatory bowel diseases (BMD), blood coagulation, and the proper functioning of the cardiovascular system. In the human body, more than $99 \%$ of Ca is stored in bones. Therefore, a decreased serum calcium level leads to its release from bones and causes bone tissue resorption [1] . Furthermore, an insufficient calcium intake causes hormonal disorders, leading to a higher risk of fractures. Calcium can be found in such sources as milk, dairy products, and green leafy vegetables ${ }^{[2]}$. Additionally, the human body contains about $700 \mathrm{~g}$ of phosphorus $(P)$, which is mainly stored in bones (80-90\%). Hence, both its excessive and inadequate intake can develop osteoporosis. Phosphorus deficiency, or its insufficient supply to calcium supply ratio, causes bone resorption and inhibits bone mineralization and bone formation. On the other hand, an oversupply of $P$, particularly with insufficient $\mathrm{Ca}$ intake, results in excessive parathormone excretion and the loss of bone mass [주.

The insufficient intake of calcium was estimated in $80-86 \%$ of IBD patients, who avoid milk and dairy products due to lactose intolerance [4] . Patients with IBD have lower calcium and phosphate levels in comparison with healthy individuals []․

Another cause of calcium malabsorption is the use of steroids as well as the occurrence of diarrhoea. The supplementation of calcium in a 1000-1500 mg/day dose is recommended for most patients with inflammatory bowel diseases. Furthermore, patients treated with steroids require calcium and vitamin D supplementation [4]. Calcium intake was correlated negatively with the femoral neck BMD but not with the lumbar spine BMD in IBD patients []․ Premenopausal women suffering from IBD consumed insufficient amounts of calcium and vitamin D, and their intake of Ca and vitamin D was correlated [ $\underline{\text { ] }}$. Moreover, a low calcium serum level was observed in patients more frequently than in the control group, although it was insignificant. Additionally, the Ca serum level was negatively correlated with steroids [ㄷ]. A meta-analysis demonstrated that calcium supplementation without other substances (for example, 
vitamin D) did not alter the risk of femoral neck fractures in both sexes []. The study revealed that an increased intake of calcium by every $300 \mathrm{mg}$ decreased the risk of fractures, although it was nonlinear. The highest risk was found in the intake below $751 \mathrm{mg}$ of calcium. The fracture risk was unchanged in the intakes of more than $1137 \mathrm{mg}$ and $882-996 \mathrm{mg}$ of calcium [9]. Gutiérrez et al. demonstrated that a one-week diet rich in phosphorus (1677 $\pm 167 \mathrm{mg} /$ day) increased Fibroblast Growth Factor 23 (FGF23), osteocalcin, and osteopontin levels. The aforementioned results suggest that a phosphorus-rich diet negatively affects health [1이, and that women over 45 years of age, both with and without osteoporosis, consume similar amounts of calcium. Thus, Ca intake was not associated with the incidence of fractures [11].

\section{Magnesium}

Magnesium ( $\mathrm{Mg}$ ) is absorbed in the small intestine, and its absorption ranges from $30 \%$ to $80 \%$. Bones store about $60 \%$ of the total body magnesium. The main sources of $\mathrm{Mg}$ are legumes, seeds, nuts, almonds, spinach, and buckwheat. Not only is this element responsible for the stability and permeability of cell membranes but it also maintains the DNA double helix integrity and regulates the activity of about 300 enzymes [12]. On the other hand, magnesium deficiency causes decreased osteoblast and osteoclast activity, resulting in bone metabolism disorders [13] . Chronic hypomagnesemia leads to the disturbance of parathyroid hormone production, leading to hypocalcaemia $[\underline{14}]$.

Patients with UC and CD consumed a lower amount of Mg than healthy adults. CD patients consumed $60-63 \%$ of the daily magnesium requirement $[\underline{15}][\underline{16}]$. Magnesium intake correlated with BMD, with a stronger correlation found in men than in women [르. Postmenopausal women who consumed $422.5 \mathrm{mg}$ and more of Mg per day presented a higher hip and total body BMD by $3 \%$ and $2 \%$, respectively, than the individuals supplying $<206.5 \mathrm{mg} \mathrm{Mg} /$ day. No association was observed between magnesium intake and the risk of fractures. On the other hand, a high magnesium dose was associated with a higher risk of forearm and wrist fractures in comparison with a low Mg intake. The authors paid attention to the subjects with a high supply of magnesium, since they reported much physical activity, which increases the frequency of falls ${ }^{[17]}$. The supplementation of magnesium $(106 \mathrm{mg})$ and calcium $(1200 \mathrm{mg})$ for 4 weeks in postmenopausal women did not change the serum parathyroid hormone level both in the study and the control group. However, the supplementation increased the serum CTX (C-terminal telopeptide) level-i.e., a bone resorption marker [18]. A conducted meta-analysis indicated that a high magnesium intake was not associated with a lower risk of hip fractures. On the other hand, magnesium dose was connected with the hip and femoral neck BMD, although no association was found with the lumbar spine BMD [1ㅁ].

\section{Sodium (Na)}

The absorption of water and electrolytes, including sodium ( $\mathrm{Na}$ ), takes place in the colon. The lymphatic function of the large intestine can be impaired in the course of the mucosal inflammation [20] .

In spite of the fact that the human body contains as much as $105 \mathrm{~g}$ of sodium, the intake of $\mathrm{Na}$ in the population is still too high, with some people consuming 9-12 g salt per day, which results in numerous

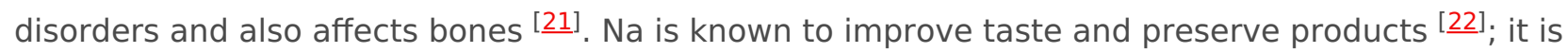
usually found in salt ( $40 \%$ of mass), meat and its preparations, grains, milk, and dairy products. This element constitutes the main extracellular cation excreted in urine and sweat. Moreover, sodium is responsible for the maintenance of the acid-base balance, cell work, and the transmission of nerve impulses. Although a normal sodium $\mathrm{Na}^{+}$level is $135-145 \mathrm{mmol}$, both too high and too low $\mathrm{Na}$ concentration levels constitute a threat to health and life. In fact, hypernatremia causes weakness, headache, vomiting, loss of appetite, weak nerve reflexes, and cardiac disorders. On the other hand, hyponatremia induces neuromuscular excitability, confusion, and cardiac arrest.

Patients with UC in remission consumed non-significantly lower sodium amounts than healthy individuals [르. The sodium intake was lower in malnourished subjects than in properly nourished patients [24]. 
The Korea National Health and Nutrition Examination Survey indicated that osteoporosis was observed more frequently in postmenopausal women consuming $\geq 4001 \mathrm{mg}$ of salt per day than in those consuming $\leq 2000 \mathrm{mg} / \mathrm{day}$. A salt intake of $\geq 5001 \mathrm{mg}$ was associated with a higher risk of osteoporosis in the femoral neck compared to the consumption of $\leq 2000 \mathrm{mg} /$ day $\left.{ }^{[25}\right]$. A sodium-rich diet (11.2 $\mathrm{g}$ of salt per day) increased calcium excretion in urine and changed the serum NTX (N-terminal telopeptide) level in comparison with a low salt intake $(3.9 \mathrm{~g})$. However, there was no significant change in the concentration of Pyr (pyridoxine) and Dpyr (deoxypyridoline) ${ }^{[26}$. A meta-analysis demonstrated that a high intake of $\mathrm{Na}$ is a factor associated with a higher risk of osteoporosis. There was no significant correlation between the amount of calcium excretion in urine and bone mineral density [2]].

A low sodium diet ( $2 \mathrm{~g}$ salt/day) for 6 months decreased calcium excretion with urine in patients who consumed $3.4 \mathrm{~g}$ or more salt per day and reduced the concentration of P1NP (propeptide of type 1 collagen). There was no significant change in the serum NTX level.

On the other hand, a low-sodium diet ( $2 \mathrm{~g}$ salt per day) of 6-month duration in persons consuming $3.4 \mathrm{~g}$ or more salt per day increased the amount of excreted calcium and the serum P1NP level. The authors did not observe any changes in the serum NTX level [28].

\section{References}

1. John A. Sunyecz; The use of calcium and vitamin D in the management of osteoporosis. Therapeutics and Clinical Risk Management 2008, 4, 827-836, 10.2147/TCRM.S3552.

2. Hyoung Moo Park; Jumi Heo; Yongsoon Park; Calcium from plant sources is beneficial to lowering the risk of osteoporosis in postmenopausal Korean women. Nutrition Research 2011, 31, 27-32, 10.1016/j.nutres.2010.12.005.

3. Agata Maziarka; Kazimierz Pasternak; Calcium and phosphorus in medicine and treatment. Journal of Elementology 2012, 18, 529-539, 10.5601/jelem.2013.18.3.16.

4. Caroline Hwang; Viveca Ross; Uma Mahadevan; Micronutrient deficiencies in inflammatory bowel disease: From A to zinc. Inflammatory Bowel Diseases 2012, 18, 1961-1981, 10.1002/ibd.22906.

5. Iwona Krela-Kaźmierczak; Aleksandra Szymczak; Maciej Tomczak; Liliana Łykowska-Szuber; Krzysztof Linke; Piotr Eder; Calcium and phosphate metabolism in patients with inflammatory bowel diseases.. Polish Archives of Internal Medicine 2015, 125, 588-590, 10.20452/pamw.2981.

6. Nayane Regina Araújo Pierote; Amanda Ferraz Braz; Susy L. Barros; José Machado Moita Neto; José Miguel L. Parente; Maria Da Cruz M. Silva; Mayara S. Beserra; Nina Rosa M. Soares; Dilina N. Marreiro; Nadir Do Nascimento Nogueira; et al. Effect of mineral status and glucocorticoid use on bone mineral density in patients with Crohn's disease. Nutrition 2018, 48, 13-17, 10.1016/j.nut.2017.10.016.

7. Charles N. Bernstein; Savita Bector; William D. Leslie; Lack of relationship of calcium and vitamin D intake to bone mineral density in premenopausal women with inflammatory bowel disease. American Journal of Gastroenterology 2003, 98, 2468-2473, 10.1111/j.1572-0241.2003.07676.x.

8. Heike A Bischoff-Ferrari; B. Dawson-Hughes; John A Baron; Peter Burckhardt; Ruifeng Li; Donna Spiegelman; Bonny Specker; John E Orav; John B Wong; Hannes B Staehelin; et al.Eilis O'reillyDouglas P KielWalter C Willett Calcium intake and hip fracture risk in men and women: a meta-analysis of prospective cohort studies and randomized controlled trials. The American Journal of Clinical Nutrition 2007, 86, 1780-1790, 10.1093/ajcn/86.5.1780.

9. Eva Warensjö; Liisa Byberg; Håkan Melhus; Rolf Gedeborg; Hans Mallmin; Alicja Wolk; Karl Michaëlsson; Dietary calcium intake and risk of fracture and osteoporosis: prospective longitudinal cohort study. BMJ 2011, 342, d1473d1473, 10.1136/bmj.d1473.

10. Orlando M. Gutiérrez; Alexandra Luzuriaga-McPherson; Yiming Lin; Linda C. Gilbert; Shin-Woo Ha; George R. Beck; Impact of Phosphorus-Based Food Additives on Bone and Mineral Metabolism.. The Journal of Clinical Endocrinology \& Metabolism 2015, 100, 4264-71, 10.1210/jc.2015-2279.

11. Patrice Fardellone; François-Emery Cotté; Christian Roux; E. Lespessailles; Florence Mercier; Anne-Françoise Gaudin; Calcium intake and the risk of osteoporosis and fractures in French women. Joint Bone Spine 2010, 77, 154-158, 10.1016/j.jbspin.2009.08.007.

12. Szymczyk, Halina; Magnesium-Essential trace element for the proper functioning of the body. Farmacja Współczesna 2016, 9, 217-223.

13. Ailsa Welch; Jane Skinner; Mary Hickson; Dietary Magnesium May Be Protective for Aging of Bone and Skeletal Muscle in Middle and Younger Older Age Men and Women: Cross-Sectional Findings from the UK Biobank Cohort. Nutrients 2017, 9, 1189, 10.3390/nu9111189.

14. Masahiro Yamamoto; Toru Yamaguchi; Mika Yamauchi; Shozo Yano; T. Sugimoto; Acute-onset hypomagnesemia- 
induced hypocalcemia caused by the refractoriness of bones and renal tubules to parathyroid hormone. Journal of Bone and Mineral Metabolism 2011, 29, 752-755, 10.1007/s00774-011-0275-7.

15. Azita Hekmatdoost; Farhad Vahid; Samaneh Rashvand; Mahya Sadeghi; The association between index of nutritional quality and ulcerative colitis: A case-control study. Journal of Research in Medical Sciences 2018, 23, 67, 10.4103/jrms.JRMS_555_17.

16. Lorian Taylor; Abdulelah Almutairdi; Nusrat Shommu; Richard Fedorak; Subrata Ghosh; Raylene A. Reimer; Remo Panaccione; Maitreyi Raman; Cross-Sectional Analysis of Overall Dietary Intake and Mediterranean Dietary Pattern in Patients with Crohn's Disease. Nutrients 2018, 10, 1761, 10.3390/nu10111761.

17. Tonya S Orchard; Joseph C Larson; Nora Alghothani; Sharon Bout-Tabaku; Jane A Cauley; Zhao Chen; Andrea Z Lacroix; Jean Wactawski-Wende; Rebecca D. Jackson; Magnesium intake, bone mineral density, and fractures: results from the Women's Health Initiative Observational Study.. The American Journal of Clinical Nutrition 2014, 99, 926-33, 10.3945/ajcn.113.067488.

18. Hilary Green; Chris Booth; Richard Bunning; Impact of supplementary high calcium milk with additional magnesium on parathyroid hormone and biochemical markers of bone turnover in postmenopausal women. Asia Pacific Journal of Clinical Nutrition 2002, 11, 268-273, 10.1046/j.1440-6047.2002.00300.x.

19. M. Farsinejad-Marj; Parvane Saneei; Ahmad Esmaillzadeh; Dietary magnesium intake, bone mineral density and risk of fracture: a systematic review and meta-analysis. Osteoporosis International 2015, 27, 1389-1399, 10.1007/s00198-015-3400-y.

20. Fotis Barkas; Evangelos Liberopoulos; Anastazia Kei; Moses Elisaf; Electrolyte and acid-base disorders in inflammatory bowel disease. Annals of Gastroenterology 2013, 26, 23-28.

21. Salt Reduction. WHO. Retrieved 2020-6-12

22. Kamila Fijorek; Mirosława Püsküllüoğlu; Dorota Tomaszewska; Roman Tomaszewski; Anna Glinka; Sebastian Polak; Serum potassium, sodium and calcium levels in healthy individuals - literature review and data analysis.. Folia medica Cracoviensia 2014, 54, 53-70.

23. Dominika Głąbska; Dominika Guzek; Gustaw Lech; Lech; Analysis of the Nutrients and Food Products Intake of Polish Males with Ulcerative Colitis in Remission. Nutrients 2019, 11, 2333, 10.3390/nu11102333.

24. Hee-Sook Lim; Hwa Jong Kim; Su Jin Hong; Soonkyung Kim; Nutrient Intake and Bone Mineral Density by Nutritional Status in Patients with Inflammatory Bowel Disease. Journal of Bone Metabolism 2014, 21, 195-203, 10.11005/jbm.2014.21.3.195.

25. Jung-Hwan Kim; Yunmi Kim; Hyun-Young Kim; Associations Between Reported Dietary Sodium Intake and Osteoporosis in Korean Postmenopausal Women: The 2008-2011 Korea National Health and Nutrition Examination Survey. Asia Pacific Journal of Public Health 2017, 29, 430-439, 10.1177/1010539517712759.

26. Birgit Teucher; Jack R Dainty; Caroline A Spinks; Gosia Majsak-Newman; David J Berry; Jurian A Hoogewerff; Robert J Foxall; Jette Jakobsen; Kevin D Cashman; Albert Flynn; et al.Susan J Fairweather-Tait Sodium and Bone Health: Impact of Moderately High and Low Salt Intakes on Calcium Metabolism in Postmenopausal Women. Journal of Bone and Mineral Research 2008, 23, 1477-1485, 10.1359/jbmr.080408.

27. Somaye Fatahi; Nazli Namazi; Bagher Larijani; Leila Azadbakht; The Association of Dietary and Urinary Sodium With Bone Mineral Density and Risk of Osteoporosis: A Systematic Review and Meta-Analysis. Journal of the American College of Nutrition 2018, 37, 522-532, 10.1080/07315724.2018.1431161.

28. Laura Carbone; Karen D. Barrow; Andrew J. Bush; M. David Boatright; Jean A. Michelson; Kathleen A. Pitts; Victorina N. Pintea; Andrew H. Kang; Mitchell A. Watsky; Effects of a low sodium diet on bone metabolism. Journal of Bone and Mineral Metabolism 2005, 23, 506-513, 10.1007/s00774-005-0621-8.

\section{Keywords}

sodium, dietarty; magnesium; calcium; phosphates; bone mineral density; osteoporosis; inflammatory bowel diseases 